NEW ZEALAND JOURNAL OF MATHEMATICS

Volume 52 (2021), 765-771

https://doi.org/10.53733/89

\title{
CONSTRUCTING UNCOUNTABLY MANY GROUPS WITH THE SAME PROFINITE COMPLETION
}

\author{
Nikolay Nikolov and Dan SEgal \\ (Received 1 March, 2021)
}

To the memory of Vaughan Jones

\begin{abstract}
Two constructions are described: one gives soluble groups of derived length 4 , the other uses groups acting on a rooted tree.
\end{abstract}

To what extent is a finitely generated residually finite (f.g.r.f.) group determined by its finite quotients? This question can be formulated in various ways, see for example $[\mathbf{G Z}$. The family of all finite quotients of a group $G$ is determined by its inverse limit, the profinite completion $\widehat{G}$; following $[\mathbf{G Z}]$ let us define the genus of an f.g.r.f. group $G$ to be the set of isomorphism classes of f.g.r.f. groups $H$ such that $\widehat{H} \cong \widehat{G}$. If $G$ is abelian, the genus is a singleton; if $G$ is nilpotent, the genus is finite, a deep result of P. F. Pickel $[\mathbf{P 1}]$; if $G$ is metabelian the genus can be countably infinite [P2].

Uncountable genera (in fact uncountably many such) were first constructed by Pyber $[\mathbf{P}]$ : in that case the finite quotients are products of distinct alternating groups. The only other examples (to our knowledge) are due to Nekrashevych $[\mathbf{N}]$ : here the finite quotients are 2-groups. (Added in proof: since the first version of this paper was submitted, a construction similar to ours in $\$ 2$ has been cleverly used by Kionke and Schesler $[\mathbf{K S}]$ to provide examples of both amenable and non-amenable groups having the same profinite completion.)

It struck us that the constructions introduced in $[\mathbf{K K N}]$ and in $[\mathbf{S}]$ could be adapted to yield uncountable genera.

Those of the first kind are soluble: indeed this is the first example of an uncountable genus of f.g. soluble groups. Our groups have derived length four. They couldn't be metabelian, like Pickel's groups, because there are only countably many f.g. metabelian groups; in fact our proof is more elementary than Pickel's approach, which depends on the theory of Picard groups. Whether a genus of f.g. soluble groups of derived length 3 could be uncountable seems an interesting question.

Those of the second kind, like Nekrashevych's groups, are branch groups. The method is easier than his, however: using perfect groups in place of 2-groups gives one cheap access to the relevant 'congruence subgroup property' (explained below).

Both constructions actually yield uncountably many distinct uncountable genera; we shall not spell this out but it is implicit in the proofs.

\section{Soluble Groups}

Let $G$ be the permutational wreath product $C_{2} \imath C_{2} \imath C_{\infty}$, a three-generator soluble group of derived length 3 . We shall use the (easy) fact that $G$ is residually finite.

The key result is 
Proposition 1. There is a family of $2^{\aleph_{0}}$ pairwise non-isomorphic, residually finite cyclic $\mathbb{Z} G$-modules all having the same finite images.

If these modules are $M_{\alpha}, \alpha \in X$, the corresponding split extensions $\Gamma_{\alpha}=M_{\alpha} \rtimes G$ all have isomorphic profinite completions. They are all quotients of

$$
\Gamma^{*}=\mathbb{Z} G \rtimes G=\mathbb{Z} \imath G ;
$$

if $M_{a}=\mathbb{Z} G / J_{\alpha}$ then $\Gamma_{\alpha} \cong \Gamma^{*} / K_{\alpha}$ where $K_{\alpha}=J_{\alpha} .1<\mathbb{Z} G \rtimes G$. For each $\alpha \in X$ the set of $\beta \in X$ such that $\Gamma_{\beta} \cong \Gamma_{\alpha}$ is countable, since there are only countably many epimorphisms from the 4-generator group $\Gamma^{*}$ to the countable group $\Gamma_{\alpha}$. It follows that the groups $\Gamma_{\alpha}, \alpha \in X$ lie in $2^{\aleph_{0}}$ isomorphism classes. Thus we may infer

Theorem 2. There are $2^{\aleph_{0}}$ pairwise non-isomorphic 4-generator residually finite soluble groups of derived length 4 with the same finite images. They are all quotients of $\mathbb{Z} \imath\left(C_{2} \succ C_{2} \succ C_{\infty}\right)$.

Let us set up some notation. Let $V$ be a vector space over $\mathbb{F}_{2}$ with basis $\left\{e_{i}, f_{i} \mid i \in \mathbb{Z}\right\}$. Let $a \in \mathrm{GL}(V)$ be the automorphism which swaps $e_{0}$ with $f_{0}$ and fixes the other basis vectors. Let $t \in \mathrm{GL}(V)$ be the automorphism such that $e_{i} t=e_{i+1}$ and $f_{i} t=f_{i+1}$ for each $i \in \mathbb{Z}$. Then $\langle a, t\rangle$ generate a copy of $C_{2} \imath C_{\infty}$ in $\operatorname{GL}(V)$ and we identify $G$ with $V \rtimes\langle a, t\rangle \leq V \rtimes \operatorname{GL}(V)$. Note that $G$ contains the elementary abelian subgroup $\left\langle a_{i} \mid i \in \mathbb{Z}\right\rangle$ where $a_{i}=a^{t^{i}} \in \operatorname{GL}(V)$ is the automorphism of order 2 which swaps $e_{i}$ with $f_{i}$ and fixes the other basis vectors.

For $\lambda \in Y:=\{0,1\}^{\mathbb{N}}$ we define the sequence $\mathbf{c}_{\lambda}=\left(c_{i}\right)_{i \in \mathbb{N}}$ by

$$
\begin{aligned}
& c_{2 n-1}=e_{n}, c_{2 n}=f_{n} \text { if } \lambda(n)=0 \\
& c_{2 n-1}=f_{n}, c_{2 n}=e_{n} \text { if } \lambda(n)=1,
\end{aligned}
$$

and an ascending chain of subgroups of $V$ by

$$
H_{\lambda, i}=\left\langle e_{0}, f_{0}, e_{-1}, f_{-1}, \ldots, e_{-i}, f_{-i}, c_{1}, \ldots, c_{i}\right\rangle .
$$

The following is then clear:

Lemma 3. (i) $V=\bigcup_{i=1}^{\infty} H_{\lambda, i}$.

(ii) For each $\alpha, \beta \in Y$ and each $n \in \mathbb{N}$ there is an element $g=g(\alpha, \beta, n) \in$ $\left\langle a_{1}, \ldots, a_{n}\right\rangle<G$ such that $H_{\alpha, i}^{g}=H_{\beta, i}$ for $i=1,2, \ldots, n$.

Fix an infinite sequence of distinct primes $\left(p_{i}\right)_{i \in \mathbb{N}}$. We now define a $\mathbb{Z} G$-module $M_{\lambda}$ for each $\lambda \in Y$. For clarity, the subscript $\lambda$ will sometimes be suppressed. Let

$$
U_{\lambda, i}=U_{i}=\mathbb{F}_{p_{i}} G /\left(H_{i}-1\right) \mathbb{F}_{p_{i}} G \cong \mathbb{F}_{p_{i}} \otimes_{\mathbb{F}_{p_{i}} H_{i}} \mathbb{F}_{p_{i}} G
$$

this is the right permutation $\mathbb{F}_{p_{i}} G$ module on the right cosets $\left\{H_{i} g \mid g \in G\right\}$ of $H_{i}$ in $G$, and we fix the module generator

$$
u_{i}=1+\left(H_{i}-1\right) \mathbb{F}_{p_{i}} G .
$$

Now $M_{\lambda}$ is defined to be the cyclic $\mathbb{Z} G$-submodule of $\prod_{i=1}^{\infty} U_{i}$ generated by $\mathbf{u}_{\lambda}=$ $\left(u_{1}, u_{2}, \ldots\right)$. Thus

$$
M_{\lambda} \cong \mathbb{Z} G / J_{\lambda}
$$


where

$$
\begin{aligned}
J_{\lambda} & =\operatorname{ann}_{\mathbb{Z} G}\left(\mathbf{u}_{\lambda}\right) \\
& =\bigcap_{i=1}^{\infty}\left(\left(H_{\lambda, i}-1\right) \mathbb{Z} G+p_{i} \mathbb{Z} G\right) .
\end{aligned}
$$

Since each $H_{i}$ is finite and $G$ is residually finite, $H_{i}$ is closed in the profinite topology of $G$. Thus the annihilator in $\mathbb{F}_{p_{i}} G$ of $u_{i}$, namely $\left(H_{i}-1\right) \mathbb{F}_{p_{i}} G$, is the intersection of finite-codimension right ideals of the form $(L-1) \mathbb{F}_{p_{i}} G$ (here $L$ ranges over the subgroups of finite index in $G$ that contain $H_{i}$ ); hence each $U_{i}$ is residually finite as a $G$-module. It follows that $\prod_{i=1}^{\infty} U_{i}$ is also a residually finite $G$-module, and then so is its submodule $M_{\lambda}$.

Lemma 4. Let $\gamma, \beta \in Y$ and let $N=N^{G} \leq V$. If $N H_{\gamma, i}=N H_{\beta, i}$ for all $i$ then the $G$-modules $M_{\gamma} / M_{\gamma}(N-1)$ and $M_{\beta} / M_{\beta}(N-1)$ are isomorphic.

Proof. It will suffice to prove the (stronger) statement

$$
J_{\gamma}+(N-1) \mathbb{Z} G=J_{\beta}+(N-1) \mathbb{Z} G .
$$

Let $x \in N$. Then for some $k$ we have $x \in H_{\gamma, i}$ and $x \in H_{\beta, i}$ for all $i>k$. Then $u_{\gamma, i}(x-1)=0$ and $u_{\beta, i}(x-1)=0$ for every $i>k$. It follows that for $\lambda=\gamma, \beta$,

$$
\mathbf{u}_{\lambda}(x-1)=\sum_{i=1}^{k} u_{\lambda, i}(x-1) \in \bigoplus_{i} U_{\lambda, i}(N-1)<\prod_{i=1}^{\infty} U_{\lambda, i} .
$$

Thus $J_{\lambda}+(N-1) \mathbb{Z} G$ maps $\mathbf{u}_{\lambda}$ into $\bigoplus_{i} U_{\lambda, i}(N-1)=D$, say.

Let $I_{\lambda}$ denote the annihilator in $\mathbb{Z} G$ of $\mathbf{u}_{\lambda}$ modulo $D$. Suppose $z \in I_{\lambda}$. Then

$$
\mathbf{u}_{\lambda} z=\left(u_{\lambda, i} s_{i}\right)_{i}
$$

with each $s_{i} \in(N-1) \mathbb{Z} G$, and $s_{j}=0$ for all $j>m$, say. By the preceding paragraph, there exists $k$ such that $u_{\lambda, i} s_{j}=0$ for each $j \leq m$ and all $i>k$. Now we choose integers $q_{i}$ such that $q_{i} \equiv \delta_{i j}\left(\bmod p_{j}\right)$ for $i, j=1, \ldots, k$. Taking $r=$ $\sum_{i=1}^{k} q_{j} s_{j}$ we have

$$
\begin{aligned}
& u_{\lambda, i} r=u_{\lambda, i} s_{i} \text { if } i \leq k \\
& u_{\lambda, i} r=0 \text { if } i>k .
\end{aligned}
$$

Thus $\mathbf{u}_{\lambda} z=\mathbf{u}_{\lambda} r$ so $z \in J_{\lambda}+r \subseteq J_{\lambda}+(N-1) \mathbb{Z} G$.

It follows that $I_{\lambda}=J_{\lambda}+(N-1) \mathbb{Z} G$. Thus it remains to show that $I_{\gamma}=I_{\beta}$.

Now let $r \in I_{\gamma}$. Then (2) and (3) hold (with $\gamma$ for $\lambda$ ), for some $k$ and some $s_{i} \in(N-1) \mathbb{Z} G$.

(3) is equivalent to

$$
r \in\left(H_{\gamma, i}-1\right) \mathbb{Z} G+p_{i} \mathbb{Z} G \quad \forall i>k .
$$

This implies

$$
r \in \bigcap_{i>k}\left((V-1) \mathbb{Z} G+p_{i} \mathbb{Z} G\right)=(V-1) \mathbb{Z} G
$$

which in turn implies that for some $k_{1}$ we have

$$
r \in\left(H_{\beta, i}-1\right) \mathbb{Z} G \forall i>k_{1} .
$$


In (2), we may enlarge $k$ arbitrarily by setting $s_{i}=0$ for finitely many values of $i>k$; so we may assume that $k \geq k_{1}$. Now (2) is equivalent to

$$
\begin{aligned}
r & \in\left(H_{\gamma, i}-1\right) \mathbb{Z} G+(N-1) \mathbb{Z} G+p_{i} \mathbb{Z} G \\
& =\left(N H_{\gamma, i}-1\right) \mathbb{Z} G+p_{i} \mathbb{Z} G \\
& =\left(N H_{\beta, i}-1\right) \mathbb{Z} G+p_{i} \mathbb{Z} G \quad(i \leq k) .
\end{aligned}
$$

Together with (4), this shows that (2) and (3) hold with $\beta$ for $\lambda$, and so $r \in I_{\beta}$. The result follows by symmetry.

Now fix $\alpha, \beta \in Y$. For any $\lambda \in Y$, every finite $G$-module image of $M_{\lambda}$ is an image of $M_{\lambda} / M_{\lambda}(N-1)$ for some subgroup $N=N^{G}$ of finite index in $V$. There exists $k$ such that $H_{\alpha, i} N=H_{\beta, i} N=V$ for all $i>k$, and there exists $g=g(\alpha, \beta, k) \in G$ such that $H_{\alpha, i}^{g}=H_{\beta, i}$ for $1 \leq i \leq k$. We can specify $\gamma \in Y$ so that $H_{\gamma, i}=H_{\alpha, i}^{g}$ for all $i$. Then $H_{\gamma, i} N=H_{\beta, i} N$ for all $i$, and Lemma 4 gives

$$
\frac{M_{\gamma}}{M_{\gamma}(N-1)} \cong \frac{M_{\beta}}{M_{\beta}(N-1)} \text {. }
$$

On the other hand,

$$
M_{\gamma} \cong \frac{\mathbb{Z} G}{J_{\gamma}}=\frac{\mathbb{Z} G}{g^{-1} J_{\alpha}} \cong \frac{\mathbb{Z} G}{J_{\alpha}} \cong M_{\alpha}
$$

It follows that $M_{\alpha} / M_{\alpha}(N-1) \cong M_{\beta} / M_{\beta}(N-1)$. We infer that $M_{\alpha}$ and $M_{\beta}$ have the same finite images as $G$-modules.

Lemma 5. The map $\lambda \longmapsto J_{\lambda}(\lambda \in Y)$ is bijective.

Proof. It suffices to show that for each $n, \lambda(n)$ is determined by $J_{\lambda}$. Now fix $n$ and set $i=2 n-1$. Then

$$
\begin{aligned}
\lambda(n)=0 & \Longleftrightarrow e_{n} \in H_{\lambda, i} \\
& \Longleftrightarrow p_{1} p_{2} \cdots p_{i-1}\left(e_{n}-1\right) \in J_{\lambda} .
\end{aligned}
$$

To see this, observe that if $g \in H_{\lambda, i}$ then $u_{\lambda, j}(g-1)=0$ for all $j \geq i$ and $p_{1} p_{2} \cdots p_{i-1} u_{j}=0$ for all $j<i$; while if $g \in G \backslash H_{\lambda, i}$ then $u_{\lambda, i} g \neq u_{\lambda, i}$ so $u_{\lambda, i} \cdot p_{1} p_{2} \cdots p_{i-1}(g-1) \neq 0$ since $p_{1} p_{2} \cdots p_{i-1}$ is invertible in $\mathbb{F}_{p_{i}}$.

Now given $\alpha \in Y$, the set of $\beta \in Y$ such that $\mathbb{Z} G / J_{\beta} \cong \mathbb{Z} G / J_{\alpha}$ is countable, since for each such $\beta$ there exists an epimorphism from $\mathbb{Z} G$ onto the countable module $\mathbb{Z} G / J_{\alpha}$ with kernel $J_{\beta}$. As $|Y|=2^{\aleph_{0}}$, Lemma 5 ensures that the modules $M_{\alpha} \cong \mathbb{Z} G / J_{\alpha}$ lie in $2^{\aleph_{0}}$ isomorphism classes, and Proposition 1 follows.

\section{Branch Groups}

For details of the following construction, see $[\mathbf{S}], \S 2$ or $[\mathbf{L S}], \S 13.4$. We start with a rooted tree $\mathcal{T}$, in which each vertex of level $n \geq 1$ has valency $1+l_{n}$ (and the root has valency $l_{0}$ ). For each $n$ we take a permutation group $T_{n}$ of degree $l_{n}$, set $W_{0}=T_{0}$, and for $n \geq 0$ let $W_{n+1}=T_{n} 2 W_{n-1}$ be the permutational wreath product. This acts in a natural way on the finite tree $\mathcal{T}[n+1]$ obtained by truncating $\mathcal{T}$ at level $n+1$. Hence the inverse limit 


$$
W=\lim _{\leftarrow} W_{n}
$$

sits naturally as a subgroup of $\operatorname{Aut}(\mathcal{T})$.

Now $W$ is a profinite group, a base for the neighbourhoods of the identity being the set of level stabilizers

$$
\operatorname{St}_{W}(n)=\operatorname{ker}(W \rightarrow \operatorname{Aut}(\mathcal{T}[n])) .
$$

A subgroup $\Gamma$ of $W$ is said to have the congruence subgroup property if the natural topology of $W$ induces the profinite topology on $\Gamma$, that is, if every subgroup of finite index in $\Gamma$ contains $\operatorname{St}_{\Gamma}(n)=\Gamma \cap \operatorname{St}_{W}(n)$ for some $n$. If this holds, then the natural homomorphism $\widehat{\Gamma} \rightarrow W$ is injective; if in addition $\Gamma$ is dense in $W$, it follows that $\widehat{\Gamma} \cong W$.

On pages 262-263 of [LS] we define four elements $\xi, \eta, a$ and $b$ of $W$, set $\Gamma=$ $\langle\xi, \eta, a, b\rangle$, and prove that under certain conditions, $\Gamma$ is both dense and satisfies the congruence subgroup property.

The conditions are as follows:

(i) $T_{n}$ is a doubly transitive subgroup of $\operatorname{Sym}\left(l_{n}\right)$ (this condition can be considerably weakened: slightly tweaking the definitions below, it suffices to asume that $T_{n}$ is transitive but not regular);

(ii) there exist a two-generator perfect group $P=\langle x, y\rangle$ and for each $n$ an epimorphism $\phi_{n}: P \rightarrow T_{n}$;

(iii) the automorphisms $\xi, \eta, a$ and $b$ are built in a particular way out of the

$$
\alpha_{n}=x \phi_{n}, \beta_{n}=y \phi_{n} \in T_{n} \leq \operatorname{Sym}\left(l_{n}\right) .
$$

Specifically, $\xi$ and $\eta$ are 'rooted automorphisms', permuting bodily the $l_{0}$ subtrees attached to the root of $\mathcal{T}$ as $\alpha_{0}, \beta_{0}$ respectively; $a$ and $b$ are so-called 'directed' (or 'spinal') automorphisms corresponding to the sequences $\left(\alpha_{n}\right),\left(\beta_{n}\right)$ - these act as rooted automorphisms on subtrees of $\mathcal{T}$ rooted at vertices one step away from a fixed infinite path (pictured on page 262 of $[\mathbf{L S}]$ ).

In principle we could choose any sequence of finite simple groups; for simplicity let us assume that $l_{n}=5$ and $T_{n}=\operatorname{Alt}(5)$ for all $n$. Put

$$
\alpha=(123), \beta=(12345) \text {. }
$$

Let $\lambda \in\{0,1\}^{\mathbb{N}_{0}}$ and set

$$
\begin{aligned}
& \alpha_{n}=\alpha, \beta_{n}=\beta \text { if } \lambda_{n}=0 \\
& \alpha_{n}=\beta, \beta_{n}=\alpha \text { if } \lambda_{n}=1 .
\end{aligned}
$$

Let $P=\operatorname{Alt}(5) \times \operatorname{Alt}(5), x=(\alpha, \beta), y=(\beta, \alpha) \in P$. Then $P=\langle x, y\rangle$, and we define $\phi_{n}: P \rightarrow T_{n}$ by

$$
\begin{aligned}
& x \phi_{n}=\alpha^{1-\lambda_{n}} \cdot \beta^{\lambda_{n}} \\
& y \phi_{n}=\alpha^{\lambda_{n}} \cdot \beta^{1-\lambda_{n}},
\end{aligned}
$$

thus $\phi_{n}$ is simply the projection of $P=\operatorname{Alt}(5) \times \operatorname{Alt}(5)$ onto either the first or the second direct factor.

Let $\Gamma(\lambda)=\langle\xi(\lambda), \eta(\lambda), a(\lambda), b(\lambda)\rangle$ denote the group $\Gamma$ constructed as above using the sequence $\lambda$. There are $2^{\aleph_{0}}$ such sequences, so we have constructed $2^{\aleph_{0}} 4$ generator subgroups of $\operatorname{Aut}(\mathcal{T})$ with profinite completion $W$. (These groups are of course residually finite since $\operatorname{Aut}(\mathcal{T})$ is.) 
Claim: For each sequence $\lambda$, the set $S(\lambda):=\{\mu \mid \Gamma(\lambda) \cong \Gamma(\mu)\}$ is countable.

The claim implies that the number of isomorphism classes among the groups $\Gamma(\lambda)$ is still $2^{\aleph_{0}}$, and yields

Theorem 6. There are continuously many pairwise non-isomorphic 4-generator residually finite groups all having the iterated wreath product $W$ as their profinite completion.

To establish the claim, we suppose that $S(\lambda)$ is uncountable, and aim to derive a contradiction.

For $\mu \in S(\lambda)$ let $\theta_{\mu}: \Gamma(\mu) \rightarrow \Gamma(\lambda)$ be an isomorphism. Then $\theta_{\mu}$ extends to a continuous automorphism $\sigma_{\mu}$ of $W$ (universal property of profinite completions).

Now the set

$$
\left\{a(\mu)^{\sigma} \mid \mu \in S(\lambda), \sigma \in \operatorname{Aut}(W)\right\} \subseteq \Gamma(\lambda)
$$

is countable because $\Gamma(\lambda)$ is a finitely generated group. Hence there exists $c \in \Gamma(\lambda)$ such that the set

$$
X:=\left\{\mu \in S(\lambda) \mid a(\mu)^{\sigma_{\mu}}=c\right\}
$$

is uncountable (all we need is: of cardinality at least 2).

One verifies easily that for each $n$,

$$
\mathrm{St}_{W}(n)=\overline{W^{e^{n}}}
$$

where $e=30$ is the exponent of $\operatorname{Alt}(5)$; thus $\operatorname{St}_{W}(n)$ is a topologically characteristic subgroup of $W$.

Let $\mu \neq \nu \in X$. Then

$$
a(\mu)^{\sigma_{\mu} \sigma_{\nu}^{-1}}=a(\nu)
$$

Now for some $n$ we have $\mu_{n} \neq \nu_{n}$. Say $a(\mu)_{n}=\alpha$ and $a(\nu)_{n}=\beta$. The continuous automorphism $\sigma_{\mu} \sigma_{\nu}^{-1}$ of $W$ fixes both $\operatorname{St}_{W}(n)$ and $\operatorname{St}_{W}(n-1)$, and therefore induces an automorphism $\tau$ on the quotient

$$
W_{n}=\operatorname{Alt}(5)^{\left(5^{n}\right)} \rtimes W_{n-1}
$$

sending the coset of $a(\mu)$ to that of $a(\nu)$ :

$$
(1, \ldots, 1, \alpha, 1,1,1,1) \cdot u \stackrel{\tau}{\longmapsto}(1, \ldots, 1, \beta, 1,1,1,1) \cdot v
$$

in an obvious notation (here, $u$ and $v$ lie in the stabilizer of the point $5^{n}-4$ ). This now implies that

$$
(1, \ldots, 1, \beta, 1,1,1,1)=\left(*, \ldots, *, \alpha^{z}, *, \ldots, *\right)
$$

for some automorphism $z$ of Alt(5). This is impossible since $\alpha$ and $\beta$ have coprime orders.

The Claim could also be quickly deduced, by a similar argument, from [LN], Prop. 8.1. Thanks to the referee for this observation. 


\section{References}

[GZ] F. Grunewald and P. Zalesskii, Genus for groups, J. Algebra 326 (2011), 130-168.

[KKN] A. Kar, P. Kropholler and N. Nikolov, On growth of homology torsion in amenable groups, Math. Proc. Camb. Phil. Soc. 162 (2017), 337-351.

[KS] S. Kionke and E. Schesler, Amenability and profinite completions of finitely generated groups, arXiv:2106.08742.

[LN] Y. Lavreniuk and V. Nekrashevych, Rigidity of branch groups acting on rooted trees, Geom. Dedicata 89 (2002), 159-179.

[LS] A. Lubotzky and D. Segal, Subgroup Growth, Birkhäuser Verlag, BaselBoston-Berlin, 2003.

[N] V. Nekrashevych, An uncountable family of 3-generated groups with isomorphic profinite completions, arXiv:1303.5782v2 [math.GR] (2013)

[P1] P. F. Pickel, Finitely generated nilpotent groups with isomorphic finite quotients, Trans. Amer. Math. Soc. 160 (1971), 327-341.

[P2] P. F. Pickel, Metabelian groups with the same finite quotients Bull. Austral. Math. Soc. 11 (1974), 115-120.

$[\mathrm{P}]$ L. Pyber, Groups of intermediate subgroup growth and a problem of Grothendieck, Duke Math. J. 121 (2004), 169-188.

[S] D. Segal, The finite images of finitely generated groups, Proc. London Math. Soc. 82 (2001), 597-613.

Mathematical Institute, University of Oxford, UK

nikolay.nikolov@maths.ox.ac.uk

All Souls College, Oxford, UK

dan.segal@all-souls.ox.ac.uk 